\title{
STUDY ON METABOLITES OF 2,5-DIMETHOXY-4-BROMAMPHETAMINE (DOB) IN HUMAN URINE USING GAS CHROMATOGRAPHY-MASS SPECTROMETRY
}

\author{
Kateřina Beránková, Marie Balíková
}

\author{
Institute of Forensic Medicine and Toxicology, $1^{\text {st }}$ Faculty of Medicine, Charles University in Prague, 12108 Prague \\ e-mail:mbali@lf1.cuni.cz
}

Received: June 10, 2005; Accepted: September 25, 2005

Key words: 2,5-Dimethoxy-4-bromamphetamine/DOB/Biotransformation/Metabolites

The study of the biotransformation of a new synthetic drug 2,5-dimethoxy-4-bromamphetamine (DOB) and identification of its metabolites in urine of a poisoned person is described using gas chromatography mass spectrometry (GC-MS) with various ways of derivatization. It has been confirmed that one of its metabolic pathways leads to the corresponding 2-O-desmethyl and 5-O-desmethyl metabolites when the latter is prevailing. It is important to know the metabolism of this neurotoxic and hallucinogenic substance as it is a prerequisite for developing reliable toxicological diagnostic procedures and for assessment of toxicological risks.

\section{INTRODUCTION}

Recently, the popularity of new illegal synthetic drugs has been accelerating among young generation in Europe and elsewhere, especially at dancing scene due to the stimulating and empatic potential of these compounds. Even though some of these drugs are scheduled among controlled substances, new derivatives are synthesized with small variation of their structure and are available on the black market usually in various tablet forms labeled with different logo symbols. Nevertheless, the true composition of illegal tablets is without any guarantee of its identity and purity and cases of serious intoxication have been reported. The case reports and experimental studies have shown that these drugs posses serious serotonergic neurotoxic properties. It is important to know metabolic pathways of these new substances which is a prerequisite for developing reliable toxicological diagnostic procedures and for assessment of toxicological risks. It can not be excluded that some pharmacological effects may be closely associated with active metabolites.

The presented contribution concerns the case of human fatal overdose with unknown substance which was identified as DOB subsequently during laboratory differential diagnostics ${ }^{1}$. Commercial immunological methods for screening appeared to be ineffective and the identification was based on mehods of GC-MS. The remaining aliquots of urine sample of this case were used for the following study of excreted metabolites.

As we know, neither human or animal metabolites of DOB have been identified to present. However, some structurally related compounds, as mescaline ${ }^{2}$ or 2,5-dimethoxy4-brom-phenethylamine ${ }^{3,4}, p$-methoxymethamphetamine ${ }^{5}$, 2,5-dimethoxy-4-ethylthiophenethylamine ${ }^{6}$ have been the subjects of previous biotransformation studies. Two competitive metabolic pathways, $\mathrm{N}$-oxidation and $\mathrm{O}$-dealkyla- tion employing different enzyme systems have been considered. The formation of phenolic metabolites by $O$-demethylation in methoxy groups and excretion into urine was substantial both in humans and in experimental animals.

The attention in our study of DOB metabolism was focused to these types of metabolites. The results of GCMS analyses of assumed metabolic compounds extracted from human urine were compared with data of synthesized metabolic standards of mono $O$-demethylated DOB in the position 2 or 5 of benzene ring. The confirmation of suppossed metabolites in urine was enabled using various types of chemical derivatization (acetylation, trifluoroacetylation, silylation) and assessing the accord in retention parameters and mass spectra.

\section{MATERIAL AND METHODS}

A remaining aliquot of human urine from a fatal case of DOB overdose was used for this study. All chemicals and solvents used were of analytical grade purity. The reference standards used for identification of the intoxicating agent were 2,5-dimethoxy-4-bromamphetamine. $\mathrm{HBr}$ was purchased from Sigma-Aldrich Prague and the presumed metabolites 2-hydroxy-5-methoxy-4-bromamphetamine and 2-methoxy-5-hydroxy-4-bromamphetamine were ordered and synthesized in Pharmaceutical Faculty of Charles University in Hradec Králové with NMR structural verification.

\section{Sample preparation:}

$4 \mathrm{ml}$ of human urine were used and processed as follows:

Without hydrolysis: $4 \mathrm{ml}$ of ethylacetate were added to urine whose $\mathrm{pH}$ value was set to 12 and extracted. Bases 


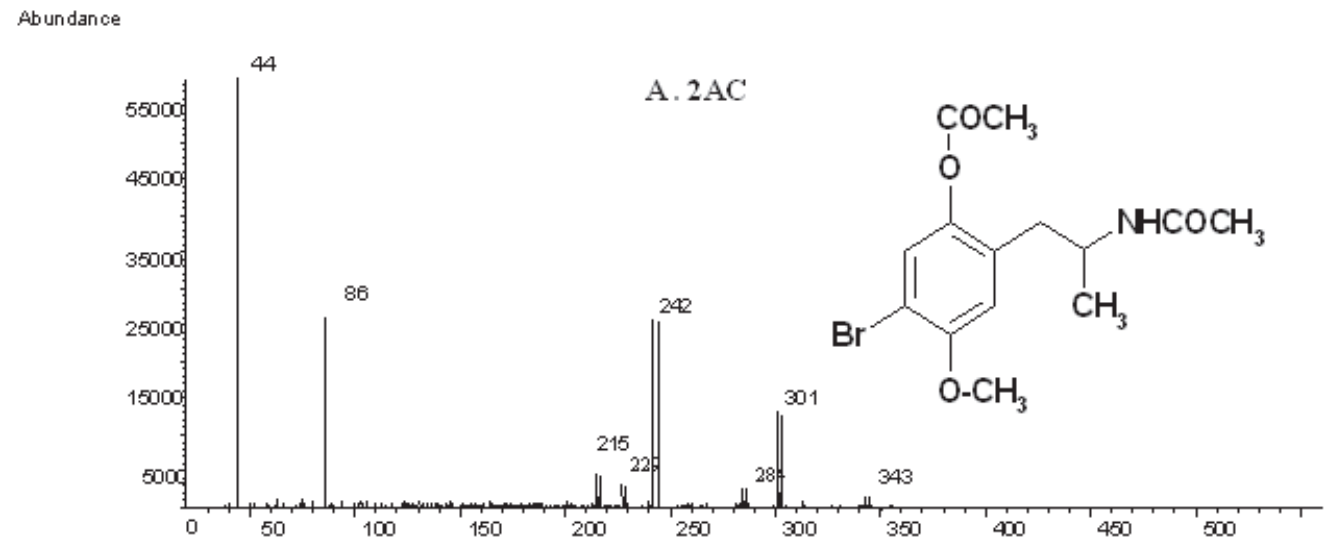

miz-s

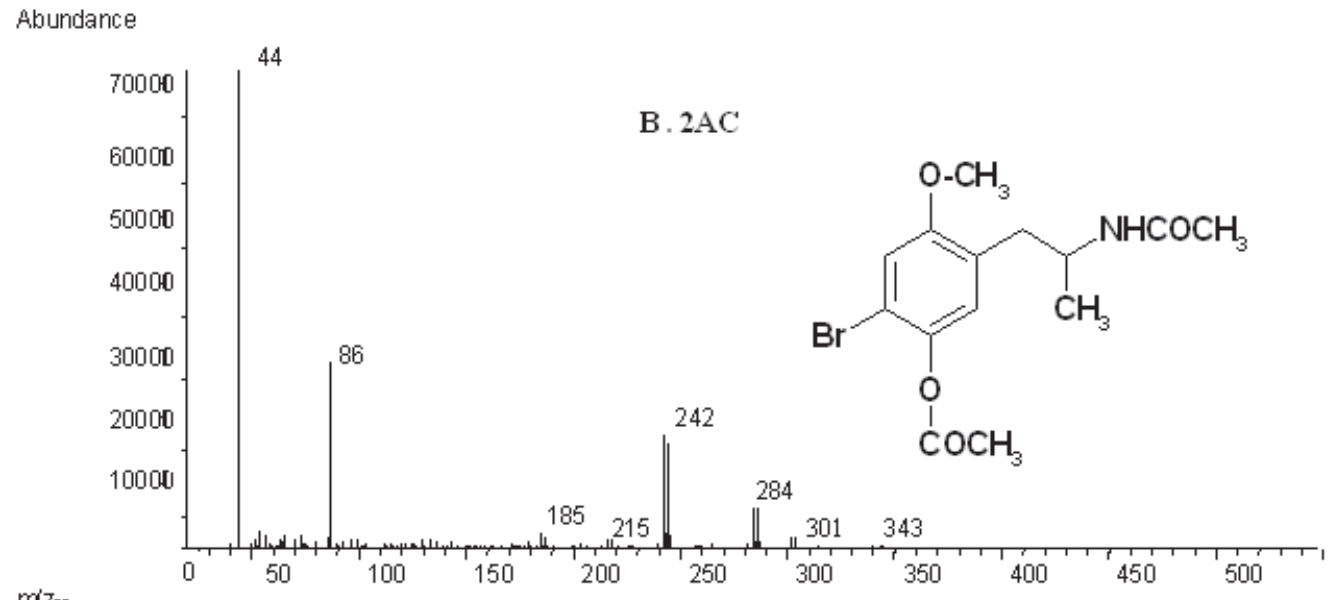

Fig. 1. Mass spectra of reference standards of metabolites DOB in acetylated form: 2-hydroxy-5-methoxy-4-bromamphetamine (A) and 2-methoxy-5-hydroxy-4-bromamphetamine (B).

were then reextracted from separated ethylacetate layer into aqueous acid and after setting $\mathrm{pH}$ to 12 again backextracted into $4 \mathrm{ml}$ of ethylacetate phase which was used for derivatization before GC-MS analysis.

With enzymatic hydrolysis: $250 \mu 1$ of $\beta$-glucuronidase (143 400 units per ml, Sigma Aldrich) and $500 \mu$ of acetate buffer ( $\mathrm{pH} 4.5)$ were added to urine with incubation at $55^{\circ} \mathrm{C}$ for 2 hours. Extraction and purification followed as above. Final ethylacetate extracts were used for derivatization before GC-MS analysis.

With acid hydrolysis: $1 \mathrm{ml}$ of concentrated $\mathrm{HCl}$ was added to urine and hydrolysis was performed at $110^{\circ} \mathrm{C}$ for an hour. After cooling, impurities were extracted into ethylacetate, the remaining aqueous layer was alkalinized ( $\mathrm{pH} \mathrm{12)} \mathrm{and} \mathrm{bases} \mathrm{were} \mathrm{extracted} \mathrm{into} \mathrm{another} 4 \mathrm{ml}$ of ethylacetate which was kept for following derivatization procedures.

\section{Derivatization:}

Acetylation: Ethylacetate extracts were derivatized with $500 \mu \mathrm{l}$ of acetic anhydride/pyridine mixture (10:1) at $60{ }^{\circ} \mathrm{C}$ for 30 minutes, then dried and reconstituted by $100 \mu \mathrm{l}$ of ethylacetate.
Trifluoroacetylation: Ethylacetate extracts were dried and derivatized with $100 \mu \mathrm{l}$ of MBTFA at $80{ }^{\circ} \mathrm{C}$ for 30 minutes.

Silylation: Ethylacetate extracts were dried and derivatized with $100 \mu \mathrm{l}$ of MSTFA at $80{ }^{\circ} \mathrm{C}$ for 30 minutes.

\section{GC-MS conditions:}

GC-MS instrument HP 6890-5973 equipped with autosampler, splitless injector, capillary column HP5-MS $30 \mathrm{~m} \times 0.25 \mathrm{~mm} \times 0.25 \mu \mathrm{m}$ were used for analysis. Carrier gas was helium at constant flow ( $1 \mathrm{ml} / \mathrm{min})$. GC temperature program was in the range of $85-250{ }^{\circ} \mathrm{C}$. Mass spectrometer was operating in EI scan mode (35-550 m/z) with standard $70 \mathrm{eV} .1 \mu \mathrm{l}$ of analyzed extract was injected at $250^{\circ} \mathrm{C}$.

\section{RESULTS}

To our present knowledge the metabolism of DOB has not been studied yet and only preliminary detection of potential metabolites in two real cases of poisoning was indicated $^{1}$. Identification of major metabolites of DOB excreted into human urine in this study was enabled partly 


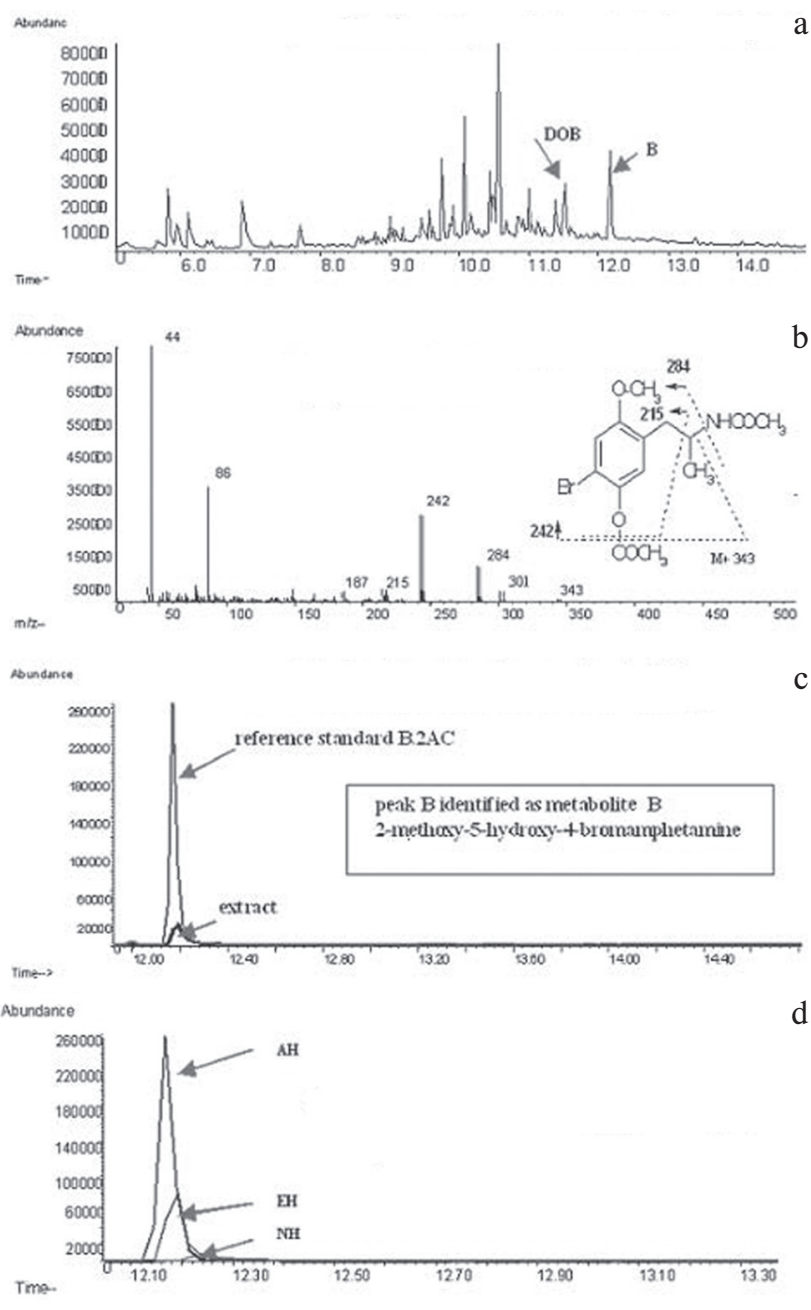

Fig. 2. GC-MS of extracts of human urine obtained after acetylation

a) Total ion chromatogram of urine extract after acid hydrolysis

b) Mass spectra of peak B (proposed metabolite B.2AC)

c) Overlay of ion chromatograms for $\mathrm{m} / \mathrm{z} 242$ for urine extract and reference standard B.2AC

d) Overlay of ion chromatograms for $\mathrm{m} / \mathrm{z} 242$ of urine extracts after different extraction procedures, comparison of total and free amount of metabolite B

$\mathrm{AH}$ - acid hydrolysis, EH - enzymatic hydrolysis,

NH - without hydrolysis

by searching for compounds containing bromine with typical mass spectra in obtained GC-MS data and partly by assuming an analogy in biotransformation of DOB with structurally related compounds where the formation of phenolic metabolites have been proved ${ }^{2-6}$.

The confirmation of suppossed metabolites of DOB excreted into urine was performed by comparison of GCMS data after three various ways of chemical derivatization (acetylation, trifluoroacetylation and silylation) and assessing accord in retention and spectral characteristics a with those of synthetised reference standards. The compared experimental data for acetylderivatives as an example are presented in the Figures 1 and 2. In Fig. 1 the mass spectra of synthetised reference metabolic standards in acetylated forms are displayed. The data in Fig. 2 encompase total ion chromatogram of urine extract after acid hydrolysis, demonstrating the presence of DOB and a presumed metabolite, mass spectra of detected potential metabolite B with proposed pathways of structural fragmentation, overlay of extracted ion chromatograms $(\mathrm{m} / \mathrm{z}$ 242) for reference monophenolic metabolite B and urine exctract after acid hydrolysis and finally the abundance of 5-O-demethylated metabolite in urine extracts after acid or enzyme hydrolysis and after basic reextraction without any previous hydrolysis step. The conjugated form of this metabolite was prevailing significantly above the free form excreted into urine. Consistent results with these findings have been confirmed also using the other two derivatization procedures.

\section{DISCUSSION}

In this work we have proved the occurence of monophenolic DOB metabolites in human urine of an intoxicated subject, one of which is formed predominantly by O-demethylation of methoxy group in the position 5 of benzene ring. Corresponding phenolic metabolites extracted from urine have been identified by comparison of the retention times and the mass spectra with synthesized reference standards using three ways of chemical derivatization as well as by searching for typical mass spectra of bromine containing compounds. It has been found that next to the parent compound, the conjugated 2-methoxy5-hydroxy-4-bromamphetamine has been prevailing metabolic form excreted into human urine after DOB overdose. No evidence of following significant $\mathrm{N}$-acetylation of this phenolic metabolite in vivo as proposed in structuraly related amines ${ }^{2-4}$ has been found using trifluoroacetylation or silylation as chemical derivatization before GC-MS. The other phenolic metabolite 2-hydroxy-5-methoxy-4bromamphetamine has been revealed only in tiny portion in urine and only using more sensitive method with trifluoroacetylation. However, the controlled experimental administration of pure substance of DOB will be the next step of our research to exclude that the batch of illicit drug taken by the subject did not contain 2-methoxy-5hydroxy-4-bromamphetamine as impurity from synthesis, even if this probability is low. There has been indication of other types of DOB metabolites excreted to human urine, however their identification is still in progress.

\section{ACKNOWLEDGEMENT}

The study has been supported by the grant of IGA MZ No. NR8332-3/2005. 


\section{REFERENCES}

1. Balíková M. (2005) Nonfatal and fatal DOB (2,5-dimethoxy-4-bromoamphetamine) overdose. Forensic Sci Int. 153, 85-91.

2. Scheline RR. Mammalian Metabolism of Plant Xenobiotics. London: Academic Press, 1978.

3. Kanamori T, Inoue H, Iwata Y, Ohmae Y, Kishi T. (2002) In vivo metabolism of 4-bromo-2,5-dimethoxyphenethylamine (2-CB) in the rat: Identification of urinary metabolites. J Anal. Toxicol 26, 61-6.
4. Carmo H, Hengstler JG. de Boer D, Ringel M, Remiao F, Carvalho F, Fernandes E, dos Reys LA. Oesch F, Bastos ML. (2005) Metabolic pathways of 4-bromo-2,5-dimethoxyphenethylamine (2C-B): analysis of phase I metabolism with hepatocytes of six species including human. Toxicology 206, 75-89.

5. Staack RF, Fehn J, Maurer HH. (2003) New designer drug p-methoxymethamphetamine: studies on its metabolism and toxicological detection in urine using gas chromatography-mass spectrometry. J. Chromatogr B 789, 27-41.

6. Li-Chan Lin, Ju-Tsung Liu, Shiu-Huey Chou, Cheng-Huang Lin (2003) Identification of 2,5-dimethoxy-4-ethylthiophenethylamine and its metabolites in the urine of rats by gas chromatography-mass spectrometry. J. Chromatogr. B 798, 241-7. 